\title{
AUTOMATIC VOCAL TRACT LANDMARK TRACKING IN RTMRI USING FULLY CONVOLUTIONAL NETWORKS AND KALMAN FILTER
}

\author{
Sasan Asadiabadi, Engin Erzin \\ Multimedia, Vision and Graphics Lab, Koç University, Istanbul, Turkey \\ E-mail: [sabadi15, eerzin]@ku.edu.tr
}

\begin{abstract}
Vocal tract (VT) contour detection in real time MRI is a pre-stage to many speech production related applications such as articulatory analysis and synthesis. In this work, we present an algorithm for robust detection of keypoints on the vocal tract in rTMRI sequences using fully convolutional networks (FCN) via a heatmap regression approach. We as well introduce a spatio-temporal stabilization scheme based on a combination of Principal Component Analysis (PCA) and Kalman filter (KF) to extract stable landmarks in space and time. The proposed VT landmark detection algorithm generalizes well across subjects and demonstrates significant improvement over the state of the art baselines, in terms of spatial and temporal errors.
\end{abstract}

Index Terms - Vocal tract dynamics. Fully convolutional networks, Heatmap regression, Kalman filter

\section{INTRODUCTION}

Study of human vocal tract dynamic plays a significant role in understanding human speech production system and many speech processing applications. Recent development in the real-time MRI provides rich information about dynamic VT shapes through speech. In this context, development of automatic algorithms to detect the landmarks defining the outlines of the vocal tract are necessary.

In one of the carliest approaches to track vocal tract boundaries in rTMRI, Bresch and Narayanan [I] presented a novel approach to segment the upper airway in a frequency domain. Their algorithm used an anatomically informed model for the vocal tract, fitting to an observed image through a gradient descent optimization procedure. Unsupervised VT boundary detection methods using Macda's gird line system [2] were proposed in [3, 4]. These algorithms simply present the vocal tract as it's lower and upper outlines, ignoring the complex structure of the tract.

Other researchers used active shape and appearance models (ASM/AAM) $[5,6]$, active contour model $(\mathrm{ACM})[7,8]$, or a combination of them [9] in a data driven detection of VT contours in the rTMRI. In other approaches the VT contour detection was formulated as a regression task. An articulator-specific Multiple Linear Regression (MLR) method was proposed by [10]. The model predicts each contour coordinates as a linear combination of pixel intensities in the region of each articulator of interest and further refines the prediction using a modified ASM method.

Beside contour based segmentation of the VT air-tissue boundaries, several regional based segmentation of the vocal tract have been proposed. [11] proposed a semantic edge detection algorithm for air-tissue boundary detection using convolutional neural networks with an encoder-decoder architecture. several another works in $[12,13,14]$, were introduced for VT contour detection using fully convolutional networks and convolutional encoder-decoder networks in a semantic segmentation fashion. However these methods were not shown to be subject independent.
In this work we formulate the VT landmark localization in an object detection problem fashion. We aim to detect several landmarks in a rtMRI frame and assign a class label to each detected landmark, according to the articulator it belongs to. We introduce a multiple output fully convolutional network to detect the VT landmarks and their articulatory class label. Upon the estimation of the landmarks at the FCN's output, a spatio-temporal stabilization technique is used to remove the possible spatial outliers and temporal jitters. We show that the proposed stabilized FCN yields in accurate subject independent VT tracking results.

\section{METHODS}

\subsection{Data Acquisition}

The data used to train the FCN model for vocal tract landmark detection in this work are acquired from USC-TIMIT database [15]. The database comprises MRI videos from the mid-sagittal view of the upper airway of 10 speakers $(5 \mathrm{~F} / 5 \mathrm{M})$, recorded at a frame rate of $23.18 \mathrm{fps}$ and a spatial resolution of $68 \times 68$ pixcls over $20 \times 20 \mathrm{~cm}$.

The method presented in [1] is utilized to generate the ground truth labels for training the FCN model. The VT landmarks generated from this method are close to the manually labeled points. However it should be noted that this method is computationally expensive and requires several minutes per frame in it's original MATLAB implementation. Figure I illustrates samples of the labeled landmarks.

\subsection{VT Landmark Localization}

A vocal tract configuration is described by a set of $M$ landmarks along the upper and lower VT profiles from the larynx to the lips, separating the tract tissue from the vocal cavity airway, as shown in Figure 1. The VT landmark localization problem is defined as the estimation of landmark coordinates along the vocal tract, in the midsagittal view of a MRI frame. It is also important to recognize the articular each landmark belongs to. Recognition of different articulators on the vocal tract is crucial in applications which involve calculating tract variables such as lip aperture, tongue tip constriction degree etc. In this work we define seven articulatory classes as 1: lower lips, 2: tongue, 3: epiglottis and trachea, 4: pharyngeal wall, 5: velum, 6: hard palate and 7: upper lips. In this framework, each VT landmark is described with two parameters: i) landmark's coordinate location, $(x, y)$; and ii) the articultory class label assigned to the landmark. Our proposed VT tracking system takes a MRI frame as input and outputs a series of landmark coordinates and their class label.

Fully convolutional networks are widely used in image segmentation problems. In this work we utilize a simplified version of $\mathrm{FCN}$ $8 \mathrm{~s}$ [16]. Heatmap regressions are one of the most popular approaches to adapt the FCN to the landmark localization problem. In this approach, the $(x . y)$ coordinates of each landmark is transformed to a 

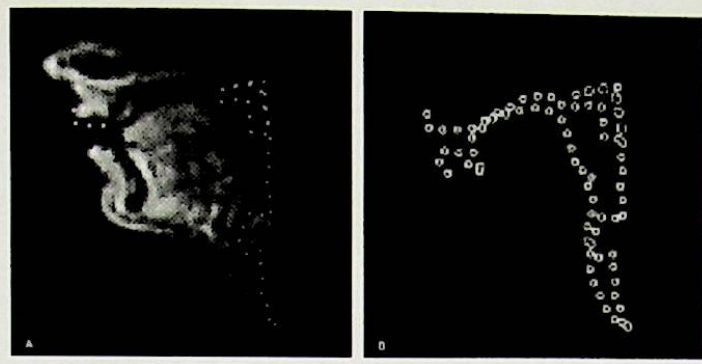

Fig. 1. A) Midsagittal MRI frame with VT landmarks color coded for different articulatory classes. B) Generated heatmaps for each landmark, supcrimposed for visualization.

separate heatmap, where the FCN learns the probability of a landmark presence at each pixel of a given image.

\subsubsection{Training dala prepration}

A key factor in a neural network is the representation of inputs and outputs of the model. In the heatmap regression approach, the FCN learns to estimate the value of a heatmap at every pixel. A one hot encoded label is assigned to each heatmap indicating the articulatory class of the corresponding landmark.

Denoting $I \in \mathbb{R}^{h \times w}$ as the MRI intensitics, normalized between $[0,1]$ in the training images set $\mathbf{I}=\left\{I_{1}, I_{2}, \ldots, I_{T}\right\}$, the true landmark coordinates and classes along the vocal tract upper and lower profile are extracted as in [1] and represented as $\mathbf{L}=$ $\left\{L_{1}, L_{2}, \ldots, L_{T}\right\}$. Here $L_{t}=\left\{\left(v_{t}^{1}, z_{t}^{1}\right),\left(v_{t}^{2}, z_{t}^{2}\right), \ldots,\left(v_{t}^{M}, z_{t}^{M}\right)\right\}$ is a series of $M$ equidistant landmarks along the VT, $v_{t}^{m}=\left[x_{t}^{m}, y_{t}^{m}\right]$ is the articulatory coordinates and $z_{t}^{m} \in\{1,2, \ldots, C\}$ is the articulatory class of $m$-th landmark in the $t$-th training sample. Note that $C$ is the number of articulatory classes, here $C=7$. It should be noted that the performance of our proposed algorithms is limited to the robustness of [1], which is used for the true label generation in this study.

The coordinates of each landmark is transformed to a heatmap, which is generated using a Gaussian kernel as

$$
H_{t}^{m}(x, y)=\frac{1}{2 \pi \sigma^{2}} \exp \left(-\frac{1}{2 \sigma^{2}}\left(\left(x-x_{t}^{m}\right)^{2}+\left(y-y_{t}^{m}\right)^{2}\right)\right),
$$

where $\sigma$ is the standard deviation and a model hyper parameter to be tuned and $H_{t}^{m} \in \mathbb{R}^{h \times w}$ is the generated heatmap for the $m$ th landmark. For the $t$-th sample in the training set, the $(x, y)$ coordinates of each landmark is transformed to a heatmap separately and stacked depth-wise to generate the output of the FCN as $\mathbf{H}_{t}=$ $\left[H_{t}^{1}, H_{t}^{2}, \ldots, H_{t}^{M}\right]$. Note that $\mathbf{H}_{t}$ is of dimension $h \times w \times M$. The landmark classes are one hot encoded and concatenated to generate articulatory class labels $\mathbf{z}_{t}=\left[z_{t}^{1}, z_{t}^{2}, \ldots, z_{t}^{M \prime}\right]$.

\subsubsection{Network architecture}

We propose a multiple output FCN to estimate the vocal tract landmarks on each articulatory region. The proposed FCN comprises a regressor to estimate a stack of heatmaps, $\hat{\mathbf{H}}_{l}$, associated with landmarks and a classifier to predict the articulatory class of each estimated landmark, $\hat{\mathbf{z}}_{t}$. Figure 2 presents architecture of the proposed FCN. As illustrated in Figure 2, the regressor and the classifier share weights at carly convolutional blocks for faster training and inference. The target outputs $\hat{\mathbf{H}}_{t}$ and $\hat{\mathbf{z}}_{t}$ are estimated separately at the regression and classification layers. The classification network consists of a number of convolutional layers which perform as a feature extractor, connected to a scrics of $M$ softmax layers predicting the class label for each landmark. The regression network on the other hand, comprises the same set of fcature extractor blocks as the classifier, connected to transpose layers to upsample the feature map size back to the input image size to estimate the stack of heatmaps. The transpose layer used in the $\mathrm{FCN}-8$ s architecture comprises learnable parameters, unlike predefined interpolation upsampling methods.

We employ MSE as the loss function for regression and cross entropy as the loss function for classification task. The network is trained to minimize a combined objective loss function defined as,

$$
L(I)=\sum_{\iota=1}^{T_{n}}\left(\frac{1}{2}\left\|\hat{\mathbf{H}}_{\iota}-\mathbf{H}_{\iota}\right\|_{2}^{2}-\frac{\alpha}{M} \sum_{m=1}^{M} \sum_{c=1}^{C} 1\left(z_{t}^{m}=c\right) \log \left(p_{t . c}^{m}\right)\right),
$$

where $1($.$) is the indicator function, T_{0}$ is the mini-batch size, and $\alpha$ is a weighting parameter. The classifier's output at the $m$-th softmax layer, $p_{t, c}^{m}$, specifies the probability that predicted landmark $\hat{v}_{t}^{m}$ belongs to class $c$ and is computed as $p_{t, c}^{m}=\exp \left(\hat{z}_{t, c}^{m}\right) / \sum_{j=1}^{C} \exp \left(\hat{z}_{t, j}^{m}\right)$

The proposed $F C N$ learns a mapping from the input MR images to the output regression and classification layers using the extracted set $\left\{I_{t},\left(\mathbf{H}_{t}, \mathbf{z}_{t}\right)\right\}_{t=1}^{T}$. We employ Adam [17] to optimize the network parameters. The model is trained with mini batch size of 128 and for 100 epochs with an adaptive learning rate reduction scheme.

\subsubsection{Heatmap to coordinate transformation}

A widely used approach to gencrate the landmark coordinates from the estimated heatmaps is to apply a channel-wisc maximum detector. However, the maximum detector may result in outlicrs. To recover the landmarks from the heatmaps robustly, we calculate each landmark as a combination of several local maximums in the estimated heatmaps. Given the estimated heatmaps $\hat{\mathbf{H}}=\left[\hat{H}^{1}, \hat{H}^{2}, \ldots, \hat{H}^{M}\right]$ at the FCN's regressor output, the landmark coordinates are recovered via finding the top $K$ maximum heatmap values as,

$$
\begin{aligned}
& \hat{v}_{1 \ldots K}^{m}=\underset{H^{\prime} \in \dot{H}^{m},\left|H^{\prime}\right|=K}{\operatorname{argmax}} \sum_{(x, y) \in H^{\prime}} \hat{H}^{m}(x, y), \\
& \hat{v}^{m}=\frac{\sum_{k=1}^{K} \hat{v}_{k}^{m} \hat{H}^{m}\left(\hat{v}_{k}^{m}\right)}{\sum_{k=1}^{K} \hat{H}^{m}\left(\hat{v}_{k}^{m}\right)}, m=1,2, \ldots, M
\end{aligned}
$$

where $\hat{v}_{1 \ldots K}^{m}$ are the locations of top $K$ maximum values in the $m$ th landmark heatmap and $\hat{v}^{m}$ is the final estimated landmark coordinate as the weighted average of these top $K$ locations. A sample heatmap estimated by the FCN is illustrated in Figure 3. Note that the heatmaps are combined via a pixel-wise hard maximum for visualization.

\subsubsection{Landmark class label smoothing}

The predicted sequence of articulatory labels $\left\{\hat{z}^{1}, \hat{z}^{2}, \ldots, \hat{z}^{M}\right\}$ at the output of the FCN may not have smooth decisions at the articulatory boundaries. Hence, we apply a median non-linear smoothing filter to clear out noisy decisions at the boundaries. The smoothed articulatory label at the $j$-th landmark is defined as $\tilde{z}^{j}=\operatorname{med}\left\{\hat{z}^{m}\right\}_{m=j-w_{s}}^{j+w_{s}}$, where med \{\} is the median filter operator and $2 w_{s}+1$ is the smoothing window size.

\subsection{VT Landmark Stabilization}

There are two major drawbacks of the proposed VT landmark tracking approach. First, although there exist a strong spatial correlation between the neighboring landmarks, they are localized indepen- 


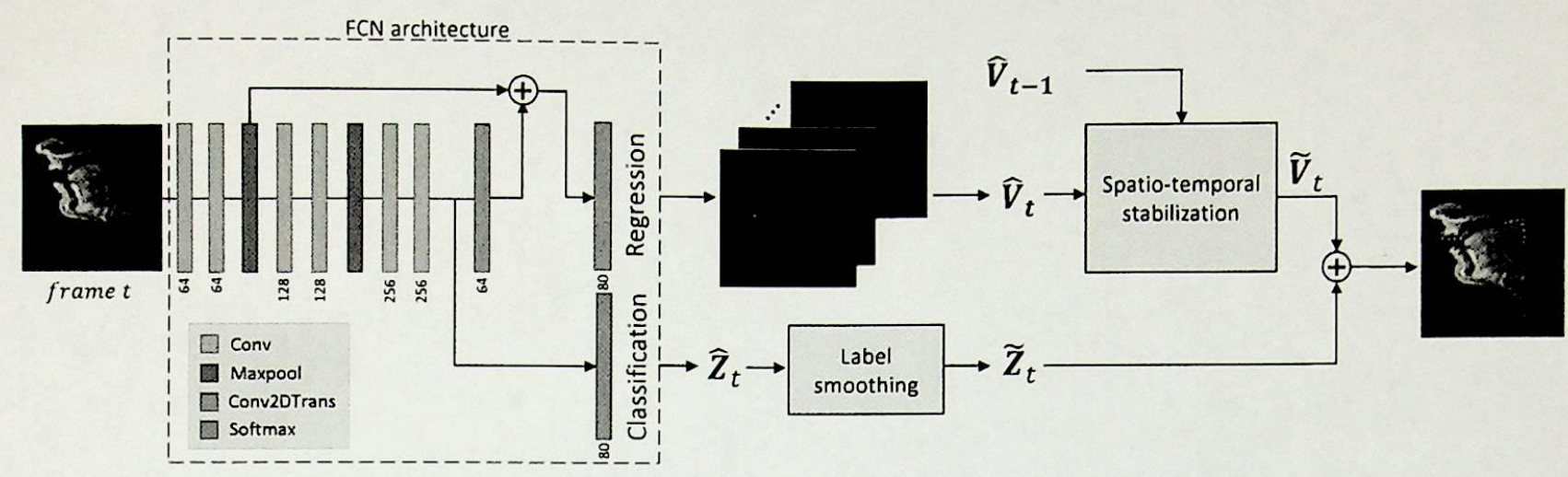

Fig. 2. Block diagram of the proposed VT landmark tracking system. Number of filters in the FCN are indicated for each layer.

dently during the regression. Second, landmark regression is executed separately for each frame that ignores temporal coherency of the articulatory movements. In order to address these two problems, we propose a stabilization scheme for the estimated VT landmarks through space and time.

Given a sequence of rtMRI frames, the FCN estimates VT landmarks for each frame independently that we can represent as $\hat{\mathbf{V}}=$ $\left\{\hat{V}_{1}, \hat{V}_{2}, \ldots, \hat{V}_{J}\right\}$, where $\hat{V}_{j} \in \mathbb{R}^{2 M \times 1}$ is the landmark vector at frame $j$. The spatio-temporal stabilization scheme receives the estimated landmark sequence and maps it to $\tilde{\mathbf{V}}=\left\{\tilde{V}_{1}, \tilde{V}_{2}, \ldots, \tilde{V}_{J}\right\}$ such that $\tilde{\mathbf{V}}$ is close to $\hat{\mathbf{V}}$ after removing spatial outliers and temporal jitter.

In the stabilization scheme, we utilize Principle Component Analysis (PCA) and Kalman filter (KF) [18]. The KF is used to stabilize the landmarks temporally from one frame to another and PCA is applied to the estimated landmarks in each frame to detect outliers and smooth the estimated VT landmarks spatially. For this purpose a shape model is generated for the landmarks from the training set $\mathbf{V}=\left\{V_{1}, V_{2}, \ldots, V_{T}\right\}$ via PCA. The VT landmark shape model is defined as the set $\mathcal{S}=\{\bar{V}, \mathbf{U}, \Lambda\}$, where $\bar{V}$ is the mean landmark shape, $\mathbf{U} \in \mathbb{R}^{2 M \times P}$ is the eigenvector of the covariance matrix $V V^{\top}$, corresponding to the $P$ largest eigenvalues and $\Lambda$ is the eigenvalue set. A new landmark shape can be generated using $V_{b}=\bar{V}+\mathbf{U} b$, where $b=\left[b^{1}, b^{2}, \ldots, b^{P}\right]^{\top}$ is the shape model parameters.

In the temporal stabilization, a landmark's stabilized location at a frame is calculated as a function of landmark's location in the previous frame (Kalman state) and it's estimated location in the current frame (observation). Rather than stabilizing each landmark location separately, we utilize the KF to stabilize VT shape parameters, which are uncorrelated representations of the landmarks. Therefore the set of estimated landmarks $\hat{\mathbf{V}}$ is transformed to the shape model parameters set $\hat{\mathbf{b}}=\left\{\hat{b}_{1}, \hat{b}_{2}, \ldots, \hat{b}_{J}\right\}$, where $\hat{b}_{j} \in \mathbb{R}^{P \times 1}$ is computed using the shape model $\mathcal{S}$, as $\hat{b}_{j}=\mathbf{U}^{\top}\left(\hat{V}_{j}-\bar{V}\right)$.

The Kalman state for the $p$-th shape parameter at frame $j$ is defined as $s_{j}^{p}=\left[b_{j}^{p}, \kappa_{j}^{p}\right]$, where $b_{j}^{p}$ is the shape parameter value and $\kappa_{j}^{p}$ models the parameter's velocity of change from one frame to another. In this experiment setup, the measurements are the estimated shape parameters $\hat{b}_{j}^{p}$. KF performs the stabilization in a cascade of two stages: i) prediction stagc, and ii) correction stage.

In the prediction stage, the state and the state estimate error covariance for the $j$-th frame is predicted using the stable state values at the previous frame as

$$
\begin{gathered}
\hat{s}_{j}^{p}=A \tilde{s}_{j-1}^{p}, \\
\hat{P}_{j}^{p}=\Lambda \tilde{P}_{j-1}^{p} \Lambda^{T}+Q,
\end{gathered}
$$

where $A$ is the state transition matrix and $Q$ is a zero mean Gaussian process noise covariance.

In the correction stage, the predicted state and state error covariance are refined using the current measurement $\hat{b}_{j}^{p}$ as

$$
\begin{gathered}
\tilde{s}_{j}^{p}=\hat{s}_{j}^{p}+K_{j}^{p}\left(\hat{b}_{j}^{p}-H \hat{s}_{j}^{p}\right), \\
\tilde{P}_{j}^{p}=\left(I-K_{j}^{p} H\right) \hat{P}_{j}^{p},
\end{gathered}
$$

where $H$ is the observation matrix mapping the state to the observations, and $K_{j}^{p}$ is called the Kalman gain and is computed as,

$$
K_{j}^{p}=\hat{P}_{j}^{p} H^{T}\left(H \hat{P}_{j}^{p} H^{T}+R\right)^{-1},
$$

where $R$ is a zero mean Gaussian measurement noise covariance. The stabilized shape parameter is then retricved from the stable statc in (7) using the observation matrix $H$ as $\tilde{b}_{j}^{p}=H \tilde{s}_{j}^{p}$.

In the spatial stabilization, the temporal stabilized shape parameters at frame $j$, are clip to the range $\left[-3 \sqrt{\lambda_{p}},+3 \sqrt{\lambda_{p}}\right]$, where $\lambda_{p}$ is the eigenvalue of the $p$-th parameter in the trained shape model $S$. Restricting the shape parameters to the defined range ensures the closeness of the estimated landmarks to the ones available in the training set, hence removes the outliers.

Upon the spatio-temporal stabilization of the shape parameters, the stable VT landmarks are then retrieved from the trained shape model $S$ as $\tilde{V}_{j}=\bar{V}+\mathbf{U} \tilde{b}_{j}$.

\section{EXPERIMENTS AND RESULT}

The ground truth VT landmarks from [1] of 3 subjects from the USCTIMIT dataset were available for training and validation in this work (subjects F1,F4,M3). We perform a leave one subject out cross validation (LOSO-CV) for all experiments. At each experiment, samples from 2 subject were used for model training and validation and the 3-rd subject was left out for testing. A number of 10 videos were randomly selected for each speaker from the dataset. During the model training, 8 videos from each of the 2 subjects were used for training and 2 videos from each were used for the validation. The performance evaluation was carried out on the 10 videos from the unseen 3-rd subject. On average, 3500 MRI samples together with their vocal tract landmark annotations were used for each subject. The FCN was implemented in Keras[19] with Tensorflow [20] backend on a NVIDIA TITAN XP GPU. 


\subsection{Bascline Methods}

We compare our proposed VT landmark tracking algorithm against some of the state of the art baselines based on their ease of implementation. Dlib is widely used in facial landmark localization tasks, where it is an implementation of Ensemble of Regression Trees (ERT) presented in [21]. Recently [22] successfully used Dlib in their work to detect vocal tract landmarks. We utilized Python implementation of Dlib to train a VT bounding box detector and landmarks predictor [23]. Multiple Linear Regression (MLR) is presented in [10] to detect vocal tract landmarks in a cascade of three linear models. Separate shape models are generated for MR image intensities and the VT contours using PCA. A linear predictor is then trained to learn the mapping between image shape parameters and the VT contour parameters. See [10] for more details. We utilized scikit-learn library in Python [24] to implement the PCA and linear regression modeling.

\subsection{Hyper Parameter Tuning}

We choose $M=80$ number of landmarks to describe the VT in a MRI frame. The performance of the proposed model is highly sensitive to the value of $\sigma$ in (1), and number of top $K$ maximums while recovering the landmark coordinates from the heatmaps in in (3). While a very small value for $\sigma$ could prevent the FCN from learning the spatial texture around each landmark, a high value could result in a noisy output. Performing a grid search analysis, the best test performance is observed for $(\sigma, K)=(3,25)$ values. The number of eigenvectors in the VT shape model $S$ is selected as $P=12$ to explain $95 \%$ of the shape variations in the training set. We choose $2 w_{s}+1=5$ for smoothing the articulatory class labels. The weighting parameter between MSE and cross entropy loss in (2) is selected as $\alpha=0.1$

\subsection{Evaluations}

We define two intraframe and interframe error metrics to evaluate the performance of the proposed VT landmark detection algorithm in space and time respectively. Mean Sum of Distance (MSD) is used as the intraframe performance assessment metric. MSD measures the difference between two contours by computing the distance between closest vertices of each contour. For a test video of length $T$ frames, given the ground label landmarks at frame $\iota$ as $V_{\iota}=$ $\left[v_{\iota}^{1}, v_{\imath}^{2}, \ldots, v_{\imath}^{M}\right]$ and their cstimated values as $\hat{V}_{t}=\left[\hat{v}_{\iota}^{1}, \hat{v}_{\iota}^{2}, \ldots, \hat{v}_{\iota}^{M}\right]$, the MSD is defined as.

$$
M S D=\frac{1}{2 T M} \sum_{t=1}^{T}\left(\sum_{i=1}^{M} \min _{j}\left\|v_{t}^{i}-\hat{v}_{t}^{j}\right\|_{2}+\sum_{i=1}^{M} \min _{j}\left\|\hat{v}_{t}^{i}-v_{t}^{j}\right\|_{2}\right)
$$

The temporal stability i.e. interframe error, measures the frame to frame change in each estimated landmark with respect to the true landmark changes, and is calculated via introducing a Landmark Temporal Stability Error (LTSE) defined as,

$$
\text { LTSE }=\frac{1}{T M} \sum_{t}^{T} \sum_{m}^{M}\left\|\left(v_{t}^{m}-v_{t-1}^{m}\right)-\left(\hat{v}_{t}^{m}-\hat{v}_{t-1}^{m}\right)\right\|_{2}
$$

To investigate the effect of the proposed stabilizer scheme in the system performance, we evaluate the performance of $\mathrm{FCN}$ and the stabilized FCN (S-FCN) separately. The average MSD and LTSE over the test videos in all LOSO-CV are shown in Table 1 . As seen in this table, the proposed heatmap regression based algorithm perform better than the baseline schemes. It is observed that the stabilization scheme improves the tracking performance remarkably.

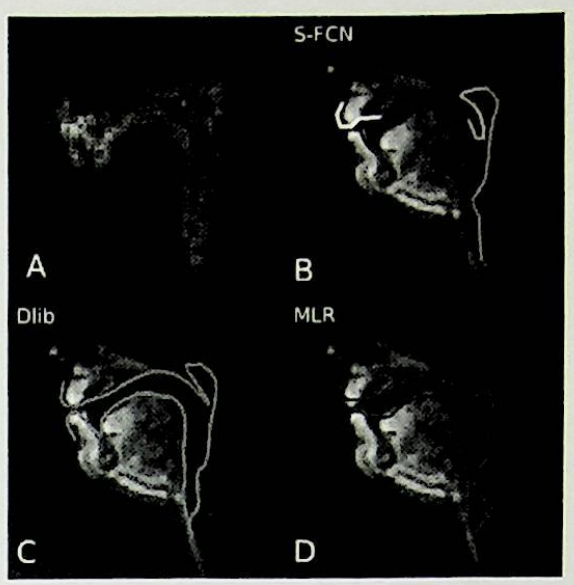

Fig. 3. A snapshot of the estimated VT landmarks on subject F5 using the proposed and the bascline methods. A: cstimated heatmap at the FCN's regression layer.

Figure 3 illustrates an example of the VT contours estimated using the proposed S-FCN scheme vs. the baselines. As obvious from this figure the proposed method accurately detects the defined articulators on the tract and generalizes better over the unseen subject as compared to the baselines.

We as well compare the different schemes with respect to the computational complexity. The average VT landmark detection time per frame is reported in Table 1. It is observed that despite the deep architecture of the FCN, the proposed method's runtime per MRI frame is quite low on a normal CPU desktop. However on a GPU the presented algorithm performs the estimation at $\sim 9$ frames per second.

Table 1. Average MSD and LTSE errors in pixels over test videos and average detection times in seconds per frame.

\begin{tabular}{lcccc}
\hline Method & MSD & LTSE & Time[CPU] & Time[GPU] \\
\hline Dlib & 5.74 & 10.46 & 0.025 & 0.016 \\
MLR & 6.56 & 8.90 & 0.04 & 0.023 \\
FCN & 5.18 & 8.85 & 1.54 & 0.120 \\
S-FCN & 4.90 & 6.75 & 1.55 & 0.123 \\
\hline
\end{tabular}

\section{CONCLUSION}

In this work we presented a robust subject independent vocal tract boundary segmentation using fully convolutional networks. The proposed system detects the location of the landmarks via heatmap regression and predicts the articulator that each landmark belongs to We introduced a spatio-temporal stabilization scheme to compensate the spatial and temporal coherency in the articulatory movement. The stabilizer refines the location of the landmarks based on their current estimation and their location in the previous frame, using Kalman filter and PCA. The proposed approach generalizes well across subjects and under different articulatory configurations. In the future work we will integrate the stabilizer scheme into the FCN network to obtain an end-to-end VT landmark localization scheme. 


\section{REFERENCES}

[1] E. Bresch and S. Narayanan, "Region segmentation in the frequency domain applied to upper airway real-time magnetic resonance imaging," IEEE Transaction on Medical Imaging, vol. 28, no. 3, 2009

[2] S. Maeda, "Compensatory articulation during speech: evidence from the analysis and synthesis of vocal tract shapes using an articulatory model," Speech Production and Modelling, pp. 131-149, 1990.

[3] M. I. Proctor, D. Bone, A. Katsamanis, and S.S. Narayanan, "Rapid semi-automatic segmentation of real-time magnetic resonance images for parametric vocal tract analysis," in Proceedings of InterSpeech, Makuhari, Japan, September 2010, pp. 1576-1579.

[4] J. Kim, N. Kumar, S. Lee, and S.S. Narayanan, "Enhanced airway-tissue boundary segmentation for real-time magnetic resonance imaging data," in Tenth international seminar on Speech Production (ISSP10), Cologne, May 2014, pp. 222 225.

[5] T. Cootes and C. Taylor, "Active shape model search using local grey-level methods: a quantitative approach," in Proc. British Machine Vision Conference, 1993, pp. 639-648

[6] S. Silva and A. Teixeira, "Unsupervised segmentation of the vocal tract from real-time mri sequences," Computer Speech and Language, vol. 33, no. 1, pp. 25-46, 2015.

[7] M. Kass, A. Witkin, and D. Terzopoulos, "Snakes: Active contour models," International Journal of Computer Vision, vol. 1, no. 4, pp. 321-331, Jan 1988.

[8] E. Bresch, J. Adams, A. Pouzet, S. Lee, D. Byrd, and S. Narayanan, "Semi-automatic processing of real-time $\mathrm{mr}$ image sequences for speech production studies," 2006.

[9] S. Asadiabadi and E. Erzin, "Vocal tract airway tissue boundary tracking for rtMRI using shape and appearance priors," in Proceedings of InterSpeech, 2017, pp. 636-640.

[10] M. Labrunie, P. Badin, D. Voit, A.A. Joseph, J. Frahm, L. Lamalle, C. Vilain, and L. Boë, "Automatic segmentation of speech articulators from real-time midsagittal mri based on supervised learning," Speech Communication, vol. 99, pp. 2746,2018

[11] K. Somandepalli, A. Toutios, and S.S. Narayanan, "Semantic edge detection for tracking vocal tract air-tissue boundaries in real-time magnetic resonance images," in Proceedings of Interspeech, August 2017.

[12] C. A. Valliappan, A. Kumar, R. Mannem, G. R. Karthik, and P. K. Ghosh, "An improved air tissue boundary segmentation technique for real time magnetic resonance imaging video using segnet," in Proc. of the 2019 IEEE International Conference on Acoustics, Speech and Signal Processing (ICASSP), 2019 , pp. 5921-5925.

[13] R. Mannem and P. K. Ghosh, "Air-tissue boundary segmentation in real time magnetic resonance imaging video using a convolutional encoder-decoder network," in Proc. of the 2019 IEEE International Conference on Acoustics, Speech and Signal Processing (ICASSP), 2019, pp. 5941-5945.

[14] C. A. Valliappan, R. Mannem, and P. K. Ghosh, "Air-tissue boundary segmentation in real-time magnetic resonance imaging video using semantic segmentation with fully convolutional networks," in Proceedings of InterSpeech, 2018, pp. 3132-3136.
[15] S.S. Narayanan, A. Toutios, V. Ramanarayanan, A. Lammert. J. Kim, S. Lec, K. Nayak, Y. Kim, Y. Zhu, L. Goldstcin, D. Byrd, E. Bresch, P. Ghosh, A. Katsamanis, and M. Proctor. "Rcal-time magnetic resonance imaging and electromagnetic articulography database for speech production research (TC)," The Journal of the Acoustical Society of America, vol. 136, no. 3, pp. 1307, 2014 .

[16] E. Shelhamer, J. Long, and T. Darrell, "Fully convolutional networks for semantic segmentation," IEEE Trans. Pattern Anal. Mach. Intell., vol. 39, no. 4, pp. 640-651, Apr. 2017.

[17] D. P. Kingma and J. Ba, "Adam: A method for stochastic optimization," CoRR, vol. abs/1412.6980, 2014.

[18] R. E. Kalman, "A new approach to linear filtering and prediction problems," Journal of Basic Engineering, vol. 82, no. I, pp. 35,1960

[19] F. Chollet et al., "Keras," https://keras.io, 2015.

[20] M. Abadi, P. Barham, J. Chen, Z. Chen, A. Davis, J. Dean, M. Devin, S. Ghemawat, G. Irving, M. Isard, M. Kudlur J. Levenberg, R. Monga, S. Moore, D. G. Murray, B. Steiner, P. Tucker, V. Vasudevan, P. Warden, M. Wicke, Y. Yu, and $X$. Zheng, "Tensorflow: A system for large-scale machine learning." in Proceedings of the 12th USENIX Conference on Operating Systems Design and Implementation, Berkeley, CA, USA, 2016, OSDI'16, pp. 265-283, USENIX Association.

[21] V. Kazemi and J. Sullivan, "One millisecond face alignment with an ensemble of regression trees," in The IEEE Conference on Computer Vision and Pattern Recognition (CVPR), June 2014.

[22] H. Takcmoto, T. Goto, Y. Hagihara, S. Hamanaka, T. Kitamura, Y. Nota, and K. Mackawa, "Specch organ contour extraction using real-time mri and machine learning method," in Proceedings of InterSpeech, 2019.

[23] D. E. King, "Dlib-ml: A machine learning toolkit," Journal of Machine Learning Research, vol. 10, pp. 1755-1758, 2009

[24] F. Pedregosa, G. Varoquaux, A. Gramfort, V. Michel, B. Thirion, O. Grisel, M. Blondel, P. Prettenhofer, R. Weiss, V. Dubourg, J. Vanderplas, A. Passos, D. Cournapeau, M. Brucher, M. Perrot, and E. Duchesnay, "Scikit-learn: Machine learning in Python," Journal of Machine Learning Research, vol. 12, pp. 2825-2830, 2011. 